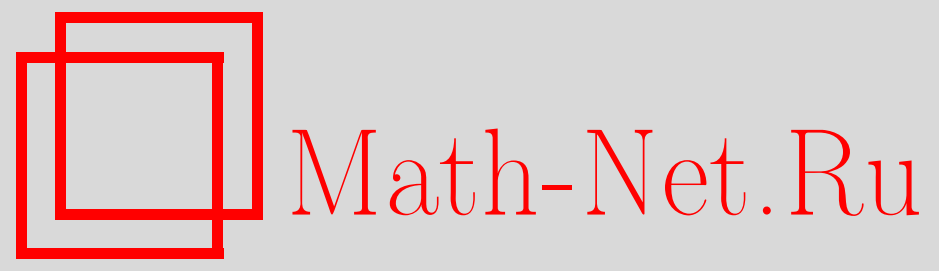

И. А. Круглов, Условия предельной равновероятности распределений в схеме линейной авторегрессии со случайным управлением на конечной группе, Дискрет. матем., 2005, том 17, выпуск $3,12-18$

DOI: https://doi.org/10.4213/dm112

Использование Общероссийского математического портала Math-Net.Ru подразумевает, что вы прочитали и согласны с пользовательским соглашением http://www . mathnet.ru/rus/agreement

Параметры загрузки:

IP : 35.173 .219 .12

26 апреля 2023 г., 14:56:06 


\section{Условия предельной равновероятности распределений в схеме линейной авторегрессии со случайным управлением на конечной группе}

() 2005 г. И. А. Круглов

Рассматривается последовательность случайных величин

$$
\mu^{(N)}=\xi_{N}\left(\mu^{(N-1)}\right)^{\zeta_{N}}, \quad N=1,2, \ldots
$$

где $\mu^{(0)}-$ случайная величина со значениями в конечной группе $G=(G, \bullet),\left(\xi_{N}, \zeta_{N}\right)$, $N=1,2, \ldots$, - последовательность одинаково распределенных случайных величин, принимающих значения в декартовом произведении $G \times \operatorname{Aut} G$, где (Aut $G$, о) - группа автоморфизмов $G$. Предполагается, что случайные величины $\mu^{(0)},\left(\xi_{N}, \zeta_{N}\right)$, $N=1,2, \ldots$, независимы. В работе найдены общие необходимые и достаточные условия того, что при произвольном распределении $\mu^{(0)}$ последовательность распределений случайных величин $\mu^{(N)}$ сходится при $N \rightarrow \infty$ к равновероятному на $G$ распределению.

Работа выполнена при поддержке программой Президента Российской Федерации подлержки ведущих научных школ, грант НШ-2358.2003.9.

Пусть $(G, \bullet)$ - конечная группа, (Aut $G$, о) - группа автоморфизмов $G, \mu^{(0)}-$ случайная величина с значениями в $G,\left(\xi_{N}, \zeta_{N}\right), N=1,2, \ldots,-$ последовательность одинаково распределенных случайных величин, принимающих значения в декартовом произведении $G \times$ Aut $G$. Будем предполагать, что случайные величины $\mu^{(0)},\left(\xi_{N}, \zeta_{N}\right), N=1,2, \ldots$, независимы, $\xi_{N}$ и $\zeta_{N}$, вообще говоря, зависимы при каждом $N=1,2, \ldots$ Построим по индукции последовательность случайных величин

$$
\mu^{(N)}=\xi_{N}\left(\mu^{(N-1)}\right)^{\zeta_{N}}, \quad N=1,2, \ldots
$$

Частные случаи последовательностей $\mu^{(N)}$ с значениями в конечных абелевых группах $G$ рассматривались ранее в работах [2]-[5], где исследовались оценки отклонения распределения $\mu^{(N)}$ от равновероятного распределения на $G$. При этом сходимость к равновероятному распределению на $G$ при $N \rightarrow \infty$ заведомо имела место в силу выбора значений пары $\left(\xi_{1}, \zeta_{1}\right)$. В [1] описано предельное поведение последовательностей некоторых условных распределений случайных величин $\mu^{(N)}$ при $N \rightarrow \infty$. В данной работе найдены общие необходимые и достаточные условия для того, чтобы при произвольном распределении $\mu^{(0)}$ последовательность распределений случайных величин $\mu^{(N)}$ сходилась при $N \rightarrow \infty$ к равновероятному на $G$ распределению. 
Из условий независимости случайных величин $\mu^{(0)},\left(\xi_{N}, \zeta_{N}\right), N=1,2, \ldots$, следует, что последовательность $\mu^{(N)}, N=0,1,2, \ldots$, есть последовательность случайных величин, связанных в простую цепь Маркова 3 с множеством состояний $G$, некоторой матрищей переходных вероятностей и начальным распределением, совпадающим с распределением $\mu^{(0)}$. Условие предельной равновероятности на $G$ распределений $\mu^{(N)}$ при $N \rightarrow \infty$ независимо от выбора распределения $\mu^{(0)}$ означает, что цепь Маркова 3 вполне регулярна (см. [6]).

Введем ряд обозначений. Для случайной величины $\eta$, принимающей значения в некотором множестве $M$, положим

$$
\Omega(\eta)=\{m \in M \mid \mathbf{P}(\eta=m)>0\} .
$$

Пусть $E$ - единичный автоморфизм, $\Omega\left(\zeta_{1}\right)=\left\{A_{1}, \ldots, A_{s}\right\}, \mathfrak{U}$ - подгруппа группы Aut $G$, порожденная множеством $\Omega\left(\zeta_{1}\right)$. Пусть $\mathfrak{N}$ - минимальный нормальный делитель группы $\mathfrak{U}$ среди таких ее нормальных делителей $L$, что $\Omega\left(\zeta_{1}\right)$ лежит в одном смежном классе группы $\mathfrak{U}$ по $L$. Положим $d=|\mathfrak{U}: \mathfrak{N}|$.

Назовем словом над алфавитом $\Omega\left(\zeta_{1}\right)$ последовательность вида

$$
z=\left(A_{i_{k}}^{\varepsilon_{k}}, \ldots, A_{i_{2}}^{\varepsilon_{2}}, A_{i_{1}}^{\varepsilon_{1}}\right), \quad i_{r} \in\{1, \ldots, s\}, \quad \varepsilon_{r}= \pm 1, \quad r=1, \ldots, k
$$

Для элемента $B \in \mathfrak{U}$ будем говорить, что слово $z$ вида (1) представляет $B$, если $B=A_{i_{k}}^{\varepsilon_{k}} \circ \ldots \circ A_{i_{1}}^{\varepsilon_{1}}$, в частности, $z$ - единичное слово, если $z$ представляет $E$. Для единичного слова $z$ пусть $l(z)=\left(\varepsilon_{1}+\ldots+\varepsilon_{k}\right)$. Нетрудно видеть, что $l(z)$ - целое число. Для $k \in\{1,2, \ldots, s\}$ пусть $\xi^{(k)}$ - случайная величина с значениями в $G$, распределение которой есть условное распределение $\xi_{1}$ при условии $\zeta_{1}=A_{k}$. Для слова $z$ вида (1) введем множество $\Omega(z) \subset G$ следующим образом. Пусть

$$
\begin{aligned}
v_{t} & =\left(A_{i_{k}}^{\varepsilon_{k}}, \ldots, A_{i_{k-t+1}}^{\varepsilon_{k-t+1}}\right), \quad t=1, \ldots, k, \\
\Omega\left(v_{1}\right) & = \begin{cases}\Omega\left(\xi^{\left(i_{k}\right)}\right), & \text { если } \varepsilon_{k}=1, \\
\left(\left(\Omega\left(\xi^{\left(i_{k}\right)}\right)\right)^{-1}\right)^{A_{i_{k}}^{-1}}, & \text { если } \varepsilon_{k}=-1,\end{cases} \\
\Omega\left(v_{t+1}\right) & = \begin{cases}\Omega\left(\xi^{\left(i_{k-t}\right)}\right)\left(\Omega\left(v_{t}\right)\right)^{A_{i_{k-t}}}, & \text { если } \varepsilon_{k-t}=1, \\
\left(\left(\Omega\left(\xi^{\left(i_{k-t}\right)}\right)\right)^{-1} \Omega\left(v_{t}\right)\right)^{A_{i_{k-t}}^{-1},} & \text { если } \varepsilon_{k-t}=-1, \quad t=1, \ldots, k-1,\end{cases}
\end{aligned}
$$

и наконец, $\Omega(z)=\Omega\left(v_{k}\right)$. Так как группа $\mathfrak{X}$ конечна, существует конечная совокупность $z_{1}, z_{2}, \ldots, z_{t}$ единичных слов в алфавите $\Omega\left(\zeta_{1}\right)$, для которой система равенств $Z_{i}=E$, $i=1, \ldots, t$, является системой определяющих соотношений группы $\mathfrak{A}$, порожденной системой образующих $\Omega\left(\zeta_{1}\right)$. Зафиксируем произвольно такую совокупность. Пусть также $z_{0}$ - произвольное единичное слово, для которого $l\left(z_{0}\right)=1$. Зафиксируем произвольно элемент $x \in \Omega\left(z_{0}\right)$.

Для каждого $k \in\{1, \ldots, s\}$ зафиксируем произвольно элемент $\sigma_{k} \in \Omega\left(\xi^{(k)}\right)$. Обозначим через $H$ минимальную подгруппу среди подгрупп $L$ группы $G$, одновременно удовлетворяющих следующим условиям:

$$
\begin{aligned}
\Omega\left(\xi^{(k)}\right) & \subset L \sigma, & k & =1, \ldots, s, \\
(L x)^{A_{k}} & =\sigma^{-1} L x \sigma_{k}, & k & =1, \ldots, s, \\
\Omega\left(z_{i}\right) & \subset L x^{l\left(z_{i}\right)}, & i & =1, \ldots, t .
\end{aligned}
$$


Пусть $G^{(1)}$ - подгруппа группы $G$, порожденная множеством $x H$, тогда $H$ - подгруппа в $G^{(1)}$, пусть $G^{(1)} / H$ - множество правых смежных классов $G^{(1)}$ по $H$. Для каждого элемента $B \in \mathfrak{U}$ определим элемент $\sigma_{B} \in G$ следующим образом: пусть $B \in A_{1}^{v} \circ \mathfrak{N}$, где $v \in\{0,1, \ldots, d-1\}, z$ - произвольное слово вида (1), представляющее $B$, тогда $\varepsilon_{1}+\ldots+\varepsilon_{k}=v+N d$ для некоторого целого $N$. Фиксируем $\tau \in \Omega(z)$ и положим $\sigma_{B}=x^{-N} \tau$.

Пусть

$$
\mathfrak{B}=\left\{B \in \mathfrak{N} \mid \sigma_{B} \in G^{(1)}\right\} .
$$

Определим отображение $\varphi: \mathfrak{B} \rightarrow G^{(1)} / H$, полагая $\varphi(B)=H \sigma_{B}$ для всех $B \in \mathfrak{B}$.

Справедливо следующее утверждение.

Теорема 1. Последовательность распределений случайных величин $\mu^{(N)}$ сходится при $N \rightarrow \infty$ к равновероятному на $G$ распределению при любом распределений $\mu^{(0)}$ тогда $u$ только тогда, когда одновременно выполняются следующие два условия: $\varphi$ сюръективно $u|\mathfrak{B}|\left|G: G^{(1)}\right|=|\mathfrak{N}|$.

Доказательство теоремы мы проведем после того, как докажем три леммы, которые справедливы независимо от выполнения условий теоремы.

Лемма 1. Подгруппа $H$ есть нормальный делитель группы $G^{(1)}$, множество $\mathfrak{B}$ есть подгруппа группы $\mathfrak{N}$, отображение $\varphi$ есть гомоморфизм группы $\mathfrak{B}$ в фактор-группу $G^{(1)} / H$.

Доказательство. Заметим, что из (4) следуют соотношения

$$
L^{A_{k}}=\sigma_{k}^{-1} L \sigma_{k}, \quad k=1, \ldots, s .
$$

Для произвольного $B \in \mathfrak{U}$ и слова $z$, представляющего элемент $B$, из соотношений (2)-(4) вытекают равенства

$$
H \Omega(z)=H \sigma=\sigma H^{B}, \quad(H x) \Omega(z)=H x \sigma=\sigma(x H)^{B}, \quad \sigma \in \Omega(z) .
$$

При $B=E$ и $z=z_{0}$ из (7) получаем равенство $H x=x H$, следовательно, $H$ нормальный делитель $G^{(1)}$.

Из соотношений (7) следует, что для всех $B \in \mathfrak{U}$

$$
G^{(1)} \sigma_{B}=\sigma_{B}\left(G^{(1)}\right)^{B} \text {. }
$$

Для слов $v, w$ обозначим $(v, w)$ слово, полученное приписыванием $w$ справа от $v$, если $w$ представляет $B$, то в силу (2)

$$
\Omega((v, w))=\Omega(w)(\Omega(v))^{B}
$$

Из соотношений (7) и (9) следуют равенства

$$
\begin{aligned}
H \sigma_{A \circ B} & =H \sigma_{B}\left(\sigma_{A}\right)^{B}, & & A, B \in \mathfrak{N}, \\
G^{(1)} \sigma_{A \circ B} & =G^{(1)} \sigma_{B}\left(\sigma_{A}\right)^{B}, & & A, B \in \mathfrak{U} .
\end{aligned}
$$

Докажем, что $\mathfrak{B}$ - подгруппа $\mathfrak{N}$. Так как группа $(\mathfrak{N}$, o) конечна, достаточно доказать, что множество $\mathfrak{B}$ замкнуто относительно групповой операции. Пусть $A, B \in \mathfrak{B}$, тогда

$$
\sigma_{A}, \sigma_{B} \in G^{(1)} \text {. }
$$


Используя соотношения (11), (8), (12), получим цепочку равенств

$$
G^{(1)} \sigma_{A \circ B}=G^{(1)} \sigma_{B}\left(\sigma_{A}\right)^{B}=\sigma_{B}\left(G^{(1)} \sigma_{A}\right)^{B}=\sigma_{B}\left(G^{(1)}\right)^{B}=G^{(1)} \sigma_{B}=G^{(1)} .
$$

Следовательно, $\sigma_{A \circ B} \in G^{(1)}$ и $A \circ B \in \mathfrak{B}$, что и требовалось доказать.

Докажем, что $\varphi$ - гомоморфизм групп. Пусть $A, B \in \mathfrak{B}$, тогда $\sigma_{A}, \sigma_{b} \in G^{(1)}$, и для некоторого натурального $k$ верно равенство

$$
H \sigma_{A}=H x^{k} .
$$

Используя соотношения (10), (7), (13), получим, что

$$
\begin{aligned}
H \sigma_{A \circ B} & =H \sigma_{B}\left(\sigma_{A}\right)^{B}=\sigma_{B}\left(H \sigma_{A}\right)^{B}=\sigma_{B}\left(H x^{k}\right)^{B} \\
& =H x^{k} \sigma_{B}=H x^{k} H \sigma_{B}=H \sigma_{A} H \sigma_{B} .
\end{aligned}
$$

Таким образом, $\varphi(A \circ B)=\varphi(A) \varphi(B)$ для всех $A, B \in \mathfrak{B}$, что и требовалось доказать. Лемма 1 доказана.

Лемма 2. Для любых $A, B \in \mathfrak{N}$ равенство $\mathfrak{B} \circ A=\mathfrak{B} \circ$ В выполняется тогда и только тогда, когда верно равенство $G^{(1)} \sigma_{A}=G^{(1)} \sigma_{B}$.

Доказательство. Для элементов $A, B \in \mathfrak{N}$ положим $C=B \circ A^{-1}$. Пусть

$$
\mathfrak{B} \circ A=\mathfrak{B} \circ B
$$

тогда из соотношение (11) и включения $C \in \mathfrak{B}$ следует, что

$$
G^{(1)} \sigma_{B}=G^{(1)} \sigma_{C \circ A}=\sigma_{A}\left(G^{(1)} \sigma_{C}\right)^{A}=\sigma_{A}\left(G^{(1)}\right)^{A}=G^{(1)} \sigma_{A} .
$$

Обратно, пусть

$$
G^{(1)} \sigma_{B}=G^{(1)} \sigma_{A}
$$

тогда из соотношения (1) следует, что

$$
\sigma_{A}\left(G^{(1)} \sigma_{C}\right)^{A}=G^{(1)} \sigma_{B}=G^{(1)} \sigma_{A}=\sigma_{A}\left(G^{(1)}\right)^{A}
$$

следовательно,

$$
G^{(1)} \sigma_{C}=G^{(1)}, \quad \sigma_{C} \in G^{(1)}, \quad B \circ A^{-1} \in \mathfrak{B}, \quad \mathfrak{B} \circ A=\mathfrak{B} \circ B .
$$

Лемма 2 доказана.

Определим подмножество $T$ элементов группы $G$, полагая

$$
T=\bigcup_{B \in \mathfrak{N}} H \sigma_{B} .
$$

Так как $\varphi$ - гомоморфизм групп, однозначно определена подгруппа $K$ группы $G^{(1)}$ такая, что $H \subset K, \varphi(\mathfrak{B})=K / H$.

Лемма 3. Множество $T$ есть объединение правых смежных классов группы $G$ по ее подгруппе $K$. Для любых $\sigma, \tau \in T$ равенство $G^{(1)} \sigma=G^{(1)} \tau$ выполняется тогда и только тогда, когда верно равенство $К \sigma=K \tau$. 
Доказательство. Докажем первое утверждение леммы. Пусть $\tau \in T, \theta \in K$, нужно доказать, что $\tau \in H \sigma_{A}, \theta \in H \sigma_{C}, H \sigma_{C}=H x^{k}$. Пусть $B=C \circ A$. Используя (7), (10), получим цепочку соотношений

$$
\theta \tau \in H \sigma_{C} H \sigma_{a}=H x^{k} \sigma_{A}=\sigma_{A}\left(H x^{k}\right)^{A}=H \sigma_{A}\left(\sigma_{C}\right)^{A}=H \sigma_{B} \subset T,
$$

то есть $\theta \tau \in T$.

Докажем второе утверждение леммы. Так как $K$ - подгруппа $G^{(1)}$, из равенства $K \sigma=K \tau$ следует, что $G^{(1)} \sigma=G^{(1)} \tau$. Обратно, пусть $G^{(1)} \sigma=G^{(1)} \tau, \sigma, \tau \in T$, введем обозначение $\theta=\sigma \tau^{-1}, \theta \in G^{(1)}$. Существуют элементы $A, B \in \mathfrak{N}$ такие, что $\sigma \in H \sigma_{B}$, $\tau \in H \sigma_{A}$. Пусть $C=B \circ A^{-1}$, тогда по лемме $1 H \sigma_{C}=H x^{k}$. Используя соотношения (7), (10), получим, что

$$
\begin{aligned}
H \theta \tau & =H \sigma=H \sigma_{B}=H \sigma_{A}\left(\sigma_{C}\right)^{A}=\sigma_{A}\left(H \sigma_{C}\right)^{A} \\
& =\sigma_{A}\left(H x^{k}\right)^{A}=H x^{k} \sigma_{A}=H \sigma_{C} \sigma_{A} \subset K \sigma_{A}=K \tau .
\end{aligned}
$$

Следовательно, $\theta \in K$ и $K \sigma=K \tau$.

Лемма 3 доказана.

Доказательство теоремы 1 . Обозначим через $e_{G}$ единичный элемент группы $G$. Как уже было отмечено выше, для выполнения условия предельной равновероятности распределения $\mu^{(N)}$ при $N \rightarrow \infty$ необходимо и достаточно, чтобы соответствующая последовательности $\mu^{(N)}$ цепь Маркова 3 была вполне регулярна. Из свойств обратимости групповых операций в $G$ и Aut $G$ следует, что матрица переходных вероятностей цепи Маркова 3 дважды стохастическая. Из общей теории цепей Маркова (см., например, [6]) следует, что условие вполне регулярности 3 будет выполнено тогда и только тогда, когда множество $G$ образует единственный класс сообщающихся состояний без циклических подклассов. При проверке последнего условия можно считать, что с вероятностью единица $\mu^{(0)}=e_{G}$, тогда для выполнения условия вполне регулярности цепи Маркова 3 необходимо, чтобы существовало $N_{0}$, для которого $\Omega\left(\mu^{(N)}\right)=G$ при всех $N>N_{0}$, и достаточно, чтобы существовало $N_{0}$, для которого $\Omega\left(\mu^{\left(N_{0}\right)}\right)=G$.

Пусть $r=\left|G^{(1)}: H\right|$. Из теоремы параграфа 4 статьи [1] следует, что существует натуральное $N_{0}$, для которого

$$
\Omega\left(\mu^{\left(k_{N}\right)}\right)=\bigcup_{B \in \mathfrak{N}} H \sigma_{B}, \quad k_{N}=r d N, \quad N>N_{0} .
$$

Суммируя сказанное выше, можно утверждать, что для выполнения условия предельной равновероятности распределения величины $\mu^{(N)}$ при $N \rightarrow \infty$ необходимо и достаточно, чтобы выполнялось равенство $G=T$.

Пусть $\mathfrak{M}$ - некоторое множество представлений правых смежных классов группы $\mathfrak{N}$ по подгруппе $\mathfrak{B}$. Тогда

$$
T=\bigcup_{B \in \mathfrak{M}} H \sigma_{B}=\bigcup_{A \in \mathbb{M}} \bigcup_{C \in \mathscr{B}} H \sigma_{C \circ A} .
$$

Из лемм 2 и 3 следует, что для всех $A \in \mathfrak{M}$

$$
\bigcup_{C \in \mathfrak{B}} H \sigma_{C \circ A}=K \sigma_{A}
$$


и что $K \circ \sigma_{A} \cap K \circ \sigma_{B}=\varnothing$ для всех $A, B \in \mathfrak{M}, A \neq B$.

Следовательно,

$$
T=\bigcup_{A \in \mathbb{M}} K \sigma_{A}
$$

есть объединение попарно не пересекающихся множеств, откуда, $|T|=|K||\mathfrak{N}: \mathfrak{B}|$. В силу леммы $2|\mathfrak{N}: \mathfrak{B}| \leqslant\left|G: G^{(1)}\right|$, кроме того, $|K| \leqslant\left|G^{(1)}\right|$. Таким образом, равенство $T=G$ выполняется тогда и только тогда, когда одновременно выполнены равенства $K=G^{(1)}$ и $|\mathfrak{N}: \mathfrak{B}|=\left|G: G^{(1)}\right|$, что равносильно условию теоремы.

Теорема доказана.

С учетом леммы 1 теорема 1 может быть переформулирована следующим образом.

Следствие 1. Последовательность распределений случайных величин $\mu^{(N)}$ сходится при $N \rightarrow \infty$ к равновероятному на $G$ распределению при любом распределении $\mu^{(0)}$ тогда $u$ только тогда, когда одновременно выполнены следующие два условия: $\varphi$ - эпиморфизм $u\left|G: G^{(1)}\right|=|\mathfrak{N}: \mathfrak{B}|$.

Относительная громоздкость условий доказанной теоремы обусловлена общностью постановки задачи. При некоторых дополнительных условиях на распределение $\left(\xi_{1}, \zeta_{1}\right)$ условия предельной равновероятности распределения $\mu^{(N)}$ удается существенно упростить. Приведем пример соответствующего утверждения (см. также [7]).

Следствие 2. Пусть множество $\Omega(\zeta)$ не лежит в одном смежном классе группы $\mathfrak{A}$ по ее собственной подгруппе, а также существует элемент $\sigma \in \Omega\left(\xi_{1}\right)$, для которого $\mathbf{P}\left(\xi_{1}=\sigma, \zeta_{1}=A\right)>0$ для всех $A \in \Omega\left(\xi_{1}\right)$. Тогда последовательность распределений случайных величин $\mu^{(N)}$ сходится при $N \rightarrow \infty$ к равновероятному на $G$ распределению при любом распределении $\mu^{(0)}$, если и полько если не существует собственной подгруппь $L$ группы $G$, удовлетворяющей соотношениям

$$
\Omega\left(\xi_{1}\right) \subset L \sigma, \quad L \sigma_{1}=\sigma_{1} L, \quad\left(L \sigma_{1}\right)^{A}=\left(L \sigma_{1}\right) \forall A \in \Omega\left(\zeta_{1}\right) .
$$

Доказательство. Докажем, что при выполнении предположений следствия подгруппа $H$ есть минимальная подгруппа группы $G$, удовлетворяющая соотношениям (15). Из условий следствия вытекает, что $\mathfrak{U}=\mathfrak{N}, d=1$, кроме того, можно считать, что $\sigma_{1}=\ldots=\sigma_{s}$. Можно также считать, что $\sigma_{A_{k}}=x^{-1} \sigma_{k}=x^{-1} \sigma_{1}$ для любого $k=\{1, \ldots, s\}$, следовательно, $G^{(1)} \sigma_{A_{k}}=G^{(1)} \sigma_{A_{j}}$ для всех $j, k=1, \ldots, s$. Но тогда по условиям следствия $\mathfrak{B}=\mathfrak{U}$. По определению подгруппы $\mathfrak{B}$ из включения $A_{1} \in \mathfrak{B}$ следует, что $\sigma_{1} \in G^{(1)}$; существует целое число $k$ такое, что $H \sigma_{1}=H x^{k}$. Из соотношений (3), (4), выполненных при $L=H$, и коммутативности фактор-группы $G^{(1)} / H$ следует, что для подгруппы $L=H$ справедливы условия (15). С другой стороны, если подгруппа $L$ группы $G$ удовлетворяет соотношениям (15), то (при условии, что $\mathbf{P}\left(\mu^{(0)}=e_{G}=1\right) \Omega\left(\mu^{(N)}\right) \subset L \sigma_{1}^{N}$ для всех $N \geqslant 1$. Из доказательства теоремы вытекает, что существует $N \geqslant 1$ такое, что $\Omega\left(\mu^{(N)}\right) \supset H$, таким образом, $L \supset H$. Итак, в условиях следствия подгруппа $H$ есть минимальная подгруппа среди подгрупп $L$ группы $G$, удовлетворяющих соотношениям (15).

Из (15) при $L=H$ следует, что для любого слова $z$ вида (1) справедливо включение $\Omega(z) \subset H \sigma_{1}^{N}$, где $N=\varepsilon_{1}+\ldots+\varepsilon_{k}$. Следовательно, $\sigma_{B} \in H$ для всех $B \in \mathfrak{A}$, $\mathfrak{B}=\mathfrak{N}=\mathfrak{A}$, а образ гомоморфизма $\varphi$ есть элемент $H$ фактор-группы $G^{(1)} / H$. Условия теоремы 1 в рассматриваемом случае эквивалентны равенствам $H=G^{(1)}=G$. С учетом приведенного выше описания подгруппы $H$ мы получаем утверждение следствия. 


\section{Список литературы}

1. Горчинский Ю. Н., Круглов И. А., Капитонов В. М., Вопросы теории распределений на конечных группах. Труды по дискретной математике (1997) 1, 85-112.

2. Егоров Б. А., Максимов Ю. И., Об одной последовательности случайных величин, принимающих значения из компактной коммутативной группы. Теория вероятностей и ее применения (1968) 13, №4, 621-630.

3. Chung F. R. K., Diaconis P., Graham R. L., A random walk problem arising in random number generation. Ann. Probab. (1987) 15, 1148-1165.

4. Diaconis P., Graham R. L., An affine walk on the hypercube. J. Comput. Appl. Math. (1992) 41, 215-235.

5. Hildebrand M., Random processes of the form $X_{n+1}=a_{n} X_{n}+b_{n}(\bmod p)$. Ann. Probab. (1993) 21, 710-720.

6. Романовский В. И., Дискретные чепи Маркова. Гостехиздат, Москва, 1949.

7. Круглов И. А., О суммировании случайных величин со значениями в конечной группе, связанных в функцию от простой однородной цепи Маркова. Обозрение прикладной и промышленной математики (2003) 10, №2, 354-355.

Статья поступила 15.12.2004. 